\title{
Validation of the Beck Depression Inventory for a Portuguese-speaking Chinese community in Brazil
}

Y.-P. Wang ${ }^{1,2}$,

L.H. Andrade ${ }^{1}$ and

C. Gorenstein ${ }^{1,3}$

\author{
${ }^{1}$ Departamento e Instituto de Psiquiatria (LIM-23), Faculdade de Medicina, \\ Universidade de São Paulo, São Paulo, SP, Brasil \\ ${ }^{2}$ Departamento de Psiquiatria, Faculdade de Medicina de Santo Amaro, \\ UNISA, São Paulo, SP, Brasil \\ ${ }^{3}$ Departamento de Farmacologia, Instituto de Ciências Biomédicas, \\ Universidade de São Paulo, São Paulo, SP, Brasil
}

\section{Correspondence \\ Y.-P. Wang \\ Instituto de Psiquiatria \\ Hospital das Clínicas, FM, USP \\ R. Dr. Ovídio P. de Campos, 785 \\ 05403-010 São Paulo, SP \\ Brasil \\ Fax: +55-11-5087-9475 \\ E-mail: gnap_inbox@hotmail.com \\ Publication supported by FAPESP.}

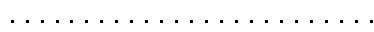

Received April 22, 2004 Accepted December 16, 2004

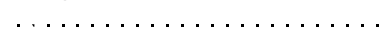

\begin{abstract}
The objective of the present study was to investigate the psychometric properties and cross-cultural validity of the Beck Depression Inventory (BDI) among ethnic Chinese living in the city of São Paulo, Brazil. The study was conducted on 208 community individuals. Reliability and discriminant analysis were used to test the psychometric properties and validity of the BDI. Principal component analysis was performed to assess the BDI's factor structure for the total sample and by gender. The mean BDI score was lower $(6.74, \mathrm{SD}=5.98)$ than observed in Western counterparts and showed no gender difference, good internal consistency (Cronbach's alpha 0.82), and high discrimination of depressive symptoms (75-100\%). Factor analysis extracted two factors for the total sample and each gender: cognitive-affective dimension and somatic dimension. We conclude that depressive symptoms can be reliably assessed by the BDI in the Brazilian Chinese population, with a validity comparable to that for international studies. Indeed, cultural and measurement biases might have influenced the response of Chinese subjects.
\end{abstract}

Key words

- Depressive symptoms

- Beck Depression Inventory

- Psychometric properties

- Validation of Depression Inventory

- Portuguese-speaking

Chinese

- Factor analysis

\section{Introduction}

As the consequence of many migrations, Chinese communities can be found in almost every country in the world. The Chinese population is the planet's largest ethnic group, comprising one fourth of the world's population, and any understanding of psychiatric disorders should take the Chinese people into account. Frequently attached to their indigenous behavioral norms and traditional cultural beliefs, Chinese immigrants are in constant change. Their real mental health status is unknown and hard to capture, and is the result of experiences of acculturation, social deprivation, uprootedness, adaptation, assimilation, Westernization, urbanization, coping style, and their own specific healthseeking behavior (1-5).

The measurement of psychopathology in cross-cultural settings has often had serious limitations. This may be especially problematic regarding self-reporting scales for mood disorders, where the understanding of the 
meaning of affect-loaded items relies on the respondent's interpretation. Reporting depressive symptoms may also be influenced by serious cultural biases in non-Western populations (e.g., language, social desirability of some behaviors), thereby resulting in poor validity. English-driven psychological advances are often adopted without any validation by researchers in different countries, and for this reason, validating psychological assessment measures with non-Anglo populations is a very important endeavor.

The original version of the Beck Depression Inventory (BDI) was introduced in 1961 (6), and its reliability and validity have been established across a broad spectrum of clinical and non-clinical populations (7). Translated into and validated in many different languages, the BDI was validated for the Chinese culture in its Chinese version (8). The Portuguese version of the BDI has proved to be reliable and valid for the Brazilian population (9-11). The aims of the present study were: 1) to assess the psychometric properties of the Portuguese version of the BDI among ethnic Chinese living in Brazil, and 2) to evaluate the validity of applying this Portuguese version of BDI for a culturally diverse sample of Chinese immigrants.

\section{Material and Methods}

\section{Subjects}

This study is part of a cross-sectional observational study (5) of non-psychotic psychopathology in a Chinese sample living in São Paulo, the most economically important city in Brazil. Community members were approached after Sunday services in a Chinese Protestant church and were asked to fill out the questionnaire. Only those with both parents of Chinese ethnicity and aged 15 years and over were considered to be eligible.

From the original sample of 214 Chinese individuals, we excluded 6 subjects because they returned incomplete questionnaires. The study sample $(\mathrm{N}=208)$ had a mean age of 24.9 (standard deviation, SD = 7.39), and included more women (57.7\%). Most individuals were single $(79.1 \%)$, students (64.5\%), Protestants (80.1\%), had university education $(87.2 \%)$, and were born in Brazil, representing second-generation Chinese $(72.0 \%)$. Fifty-six individuals $(28.0 \%)$ had emigrated from China (first-generation immigrants), about 21.13 years before the study ( $\mathrm{SD}=7.75$ ). Most of the individuals in this sample consisted of bilingual subjects speaking both Portuguese and Chinese.

\section{BDI}

The BDI is a 21-item self-report rating inventory that measures symptoms and characteristic attitudes of depression in the previous two weeks (6); the severity of each individual item is scored from 0 to 3 . There is reliability and validity evidence for both the Portuguese version (9-11) and the Chinese version $(1,4,8,12-15)$. For this Portuguesespeaking Chinese sample we employed the Portuguese version of the BDI.

\section{Statistical analysis}

The scores for the BDI items were compared by gender using the independentsample $t$-test. The internal consistency for BDI was calculated by Cronbach's alpha coefficient. Item-total correlation was evaluated to identify which items were associated more with the BDI total score.

The psychometric properties of the BDI were compared by gender and level of depressive symptomatology according to Kendall's (16) cut-off for non-clinical populations: "non-depressed" subgroup, $\mathrm{BDI} \leq 15$; "dysphoria" subgroup, $16 \leq \mathrm{BDI} \leq 20$; "depressed" subgroup, BDI $>20$. Individual item means were compared by the Student $t$-test with Bonferroni adjustments of $\mathrm{P}=0.05$ for the 21 comparisons in order to protect for family-wise error rate (individual significant 
values, $\mathrm{P}<0.002$ ).

Stepwise discriminant analysis models were applied to test: 1) if the possibly "depressed" and "non-depressed" subgroups could be separated according to all individual items, and 2) if these subgroups could be separated according to depression-specific and -nonspecific items, defined as follows: specific items - sadness, pessimism, sense of failure, guilt feelings, self-dislike, suicidal thoughts, and weight loss; nonspecific items - work inhibition, sleep disturbance, fatigability, and loss of libido (17).

Before performing factor analysis, we inspected the correlation matrix to check the strength of the correlation, and then we tested factorability using the Kaiser-Meyer-Olkin (KMO) measure for sampling adequacy and Bartlett's test of sphericity. Principal component analysis (PCA) with varimax rotation was performed using the scree test as a factor retention criterion. The statistical package SPSS was used for the analysis.

\section{Results}

\section{BDI item endorsement and internal consistency}

Item consistency analysis was carried out for the BDI raw scale by correlating item scores with total scale score. The corrected item-total correlation coefficient between each item score and the total BDI scores ranged from 0.11 to 0.58 . In Table 1 , itemtotal correlations for the total sample were higher $(>0.5)$ for items $3,4,5,7$, and 17. In contrast, items 13, 18 and 19, i.e., indecisiveness, loss of appetite and weight loss, respectively, had a correlation coefficient of $r<0.3$ with the total scale. Additionally, inter-item consistency values (Cronbach's alpha) of $0.818,0.818,0.821$ were obtained for the total sample and female and male subgroups.

Table 1 also shows the overall mean BDI scores and SD for the total sample and ac- cording to gender. Gender differences were not significant, with all mean scores for individual BDI items being similar for both genders $(\mathrm{P}>0.05)$. There was no significant difference in total scores for males (mean \pm $\mathrm{SD}=6.88 \pm 5.97)$ or females $(6.64 \pm 6.01, t$ $=-0.291$, d.f. $=206, \mathrm{P}=0.77)$. The magnitude of the difference in the means was very small (eta-squared $=0.0004$ ).

Severe depression $($ BDI $>20)$ was observed in $4.8 \%(\mathrm{~N}=10)$ of the sample as a whole, dysphoria (BDI between 16 and 20) was observed in 11 subjects $(5.3 \%)$, and the remaining $89.9 \%$ who scored 15 or less on the BDI scale were non-depressive. Considering the sample by gender, $4.1 \%$ of women $(\mathrm{N}=5)$ had scores compatible with depression, in contrast to $5.6 \%$ of men $(\mathrm{N}=5)$. The depressed subgroup showed significantly higher scores than the non-depressed sub-

Table 1. Item-total correlations for the total sample and scores for individual Beck Depression Inventory (BDI) items for the total sample and according to gender.

\begin{tabular}{|c|c|c|c|c|}
\hline \multirow[t]{2}{*}{ Item } & \multirow{2}{*}{$\begin{array}{l}\text { Item-total } \\
\text { correlation }\end{array}$} & \multirow{2}{*}{$\frac{\text { Total }}{\text { Mean } \pm \text { SD }}$} & \multirow{2}{*}{$\begin{array}{c}\text { Women } \\
\text { Mean } \pm \text { SD }\end{array}$} & \multirow{2}{*}{$\frac{\text { Men }}{\text { Mean } \pm S D}$} \\
\hline & & & & \\
\hline 1. Sadness & 0.31 & $0.67 \pm 0.53$ & $0.72 \pm 0.54$ & $0.60 \pm 0.51$ \\
\hline 2. Pessimism & 0.36 & $0.32 \pm 0.51$ & $0.30 \pm 0.53$ & $0.34 \pm 0.50$ \\
\hline 3. Sense of failure & 0.57 & $0.35 \pm 0.64$ & $0.41 \pm 0.70$ & $0.26 \pm 0.55$ \\
\hline 4. Lack of satisfaction & 0.55 & $0.44 \pm 0.72$ & $0.41 \pm 0.68$ & $0.48 \pm 0.77$ \\
\hline 5. Guilt feelings & 0.57 & $23 \pm 0.60$ & $0.20 \pm 0.64$ & $0.26 \pm 0.53$ \\
\hline 6. Sense of punishment & 0.32 & $0.27 \pm 0.66$ & $0.28 \pm 0.71$ & $0.26 \pm 0.59$ \\
\hline 7. Self-dislike & 0.58 & $0.38 \pm 0.73$ & $0.34 \pm 0.72$ & $0.44 \pm 0.74$ \\
\hline 8. Self-accusations & 0.43 & $0.42 \pm 0.73$ & $0.38 \pm 0.71$ & $0.47 \pm 0.75$ \\
\hline 9. Suicidal wishes & 0.31 & $0.06 \pm 0.29$ & $0.05 \pm 0.32$ & $0.06 \pm 0.25$ \\
\hline 10. Crying spells & 0 . & $0.35 \pm 0.67$ & $0.31 \pm 0.74$ & $0.40 \pm 0.70$ \\
\hline 11. Irritability & & $0.48 \pm 0.75$ & $0.48 \pm 0.63$ & $0.48 \pm 0.78$ \\
\hline 12. Social withdrawal & & $0.21 \pm 0.43$ & $0.18 \pm 0$ & $0.26 \pm 0.49$ \\
\hline 13. Indecisiven & 0. & $0.16 \pm 0$ & $0.19 \pm c$ & $0.12 \pm 0.36$ \\
\hline 14. Distortion of body image & 0.31 & $0.17 \pm 0.50$ & $0.20 \pm 0.55$ & $0.13 \pm 0.43$ \\
\hline 15. Work inhibition & 0.48 & $0.33 \pm 0.68$ & $0.28 \pm 0.65$ & $0.40 \pm 0.73$ \\
\hline 16. Sleep disturbance & 0.32 & $0.50 \pm 0.79$ & $0.52 \pm 0.80$ & $0.48 \pm 0.78$ \\
\hline 17. Fatigability & 0.53 & $0.46 \pm 0.69$ & $0.48 \pm 0.73$ & $0.44 \pm 0.64$ \\
\hline 18. Loss of appeti & 0.11 & $0.20 \pm 0.47$ & $0.15 \pm 0.42$ & $0.28 \pm 0.52$ \\
\hline 19. Weight loss & 0.1 & $0.29 \pm 0.68$ & $0.25 \pm 0.61$ & $0.34 \pm 0.77$ \\
\hline 20. Somatic preoccupation & 0.35 & $0.18 \pm 0.45$ & $0.19 \pm 0.47$ & $0.17 \pm 0.43$ \\
\hline 21. Loss of libido & 0.36 & $0.18 \pm 0.51$ & $0.26 \pm 0.56$ & $0.12 \pm 0.45$ \\
\hline Total & & $6.74 \pm 5.98$ & $6.64 \pm 6.01$ & $6.88 \pm 5.97$ \\
\hline Cronbach's alpha & & 0.818 & 0.818 & 0.821 \\
\hline
\end{tabular}

Data are reported as means \pm SD for $N=208$. 
group for almost all individual items. The item "fatigability" was highly reported by both the non-depressed subgroup and the depressed subgroup. Self-dislike, sense of failure, lack of satisfaction, and guilt feelings had high scores in the depressed subgroup, while the non-depressed subgroups reported more sadness, sleep disturbance, and irritability. The lowest scores for the depressed subgroups were on the items loss of appetite, weight loss, indecisiveness, and somatic preoccupation, while the non-depressed subgroup reported less suicidal wishes.

Discriminant analysis considering all BDI items showed $97.4 \%$ correct classification of non-depressed subjects, $100 \%$ of de- pressed subjects and $97.6 \%$ of the total sample. The most powerful discriminating items in ascending order were: $5,7,3,15$, 21 , and 10 , and the least important item was 19.

Discriminant analysis according to specific depression items revealed a $99.0 \%$ correct classification for the non-depressed subgroup, $100 \%$ for depressed subjects and $99.0 \%$ for the total sample. The most important items were 5, 7, and 3, and the least important was item 19. According to nonspecific depression items, there was $97.4 \%$ of correct classification for the non-depressed sub-group, $75.0 \%$ for depressed subjects and $95.7 \%$ for the total sample. The most important items were 15, 21 and 17.

Table 2. Factor loading for the Beck Depression Inventory (BDI) after varimax rotation of the total sample (N $=208$ ) and according to gender.

\begin{tabular}{|c|c|c|c|c|c|c|}
\hline \multirow[t]{2}{*}{ BDI item } & \multicolumn{2}{|c|}{ Total sample ( $\mathrm{N}=208)$} & \multicolumn{2}{|c|}{ Females ( $N=120$ ) } & \multicolumn{2}{|c|}{ Males $(\mathrm{N}=88)$} \\
\hline & Factor 1 & Factor 2 & Factor 1 & Factor 2 & Factor 1 & Factor 2 \\
\hline 1. Sadness & 0.34 & & & 0.31 & 0.33 & \\
\hline 2. Pessimism & 0.57 & & 0.58 & & 0.64 & \\
\hline 3. Sense of failure & 0.64 & & 0.47 & 0.44 & 0.79 & \\
\hline 4. Lack of satisfaction & 0.59 & & 0.58 & 0.30 & 0.44 & 0.50 \\
\hline 5. Guilt feelings & 0.66 & & 0.50 & 0.49 & 0.77 & \\
\hline 6. Sense of punishment & 0.59 & & 0.50 & & 0.67 & \\
\hline 7. Self-dislike & 0.64 & & 0.55 & 0.38 & 0.64 & \\
\hline 8. Self-accusations & 0.41 & 0.35 & 0.41 & 0.43 & & 0.44 \\
\hline 9. Suicidal wishes & 0.61 & & 0.67 & & 0.60 & \\
\hline 10. Crying spells & 0.44 & & 0.52 & & 0.40 & 0.30 \\
\hline 11. Irritability & & 0.33 & 0.36 & & & 0.61 \\
\hline 12. Social withdrawal & 0.46 & & 0.46 & & 0.41 & \\
\hline 13. Indecisiveness & & 0.45 & & 0.35 & & \\
\hline 14. Distortion of body image & & 0.61 & & 0.65 & 0.45 & \\
\hline 15. Work inhibition & 0.31 & 0.61 & & 0.77 & 0.54 & \\
\hline 16. Sleep disturbance & 0.30 & & 0.53 & & & 0.71 \\
\hline 17. Fatigability & & 0.66 & & 0.66 & 0.40 & 0.53 \\
\hline 18. Loss of appetite & & 0.37 & & & & 0.56 \\
\hline \multicolumn{7}{|l|}{ 19. Weight loss } \\
\hline 20. Somatic preoccupation & & 0.62 & & 0.60 & & 0.64 \\
\hline 21. Loss of libido & 0.33 & 0.31 & & 0.50 & 0.38 & \\
\hline$\%$ Variance explanation* & $23.33 \%$ & $8.12 \%$ & $23.50 \%$ & $9.67 \%$ & $24.81 \%$ & $10.34 \%$ \\
\hline Cronbach's alpha & 0.785 & 0.670 & 0.758 & 0.728 & 0.806 & 0.701 \\
\hline
\end{tabular}




\section{Principal component analysis}

The factorability of the total sample and female and male subgroups was accounted for by checking the strength of the relationships among variables. Inspection of the correlation matrix revealed the presence of many coefficients $\geq 0.3$. The KMO value was 0.80 , $0.72,0.71$ for the total sample and the female and male subgroups, respectively. Similarly, the Bartlett test of sphericity was statistically significant $(\mathrm{P}<0.0001)$, supporting a high strength of the relationship among variables. Therefore, all groups presented data suitable for factor analysis.

Although the PCA revealed the presence of seven components with an eigenvalue exceeding 1.0, Cattell's scree test recommended extracting only two components. For the total sample, the two-factor solution explained a total of $31.45 \%$ of variance, with the first factor accounting for $23.33 \%$ and the second for an additional $8.12 \%$ of variability. Factor loading greater than 0.40 was retained in a factor. To aid in the interpretation of these two components, varimax rotation was performed. The rotated component matrix (Table 2) revealed the presence of a simple structure, with both components showing a number of salient loadings, and variables loaded substantially on only one of components. For factor 1, the following items presented high loadings: $2,3,4,5,6,7,8,9$, 10 , and 12 , and for factor 2 items $13,14,15$, 17, and 20. Cronbach's alpha coefficients for the subscales based on the items related to factors 1 and 2 were 0.785 and 0.670 , respectively. Factor 1 represented the cognitive-affective dimension, while factor 2 represented items more related to a somaticnonspecific dimension (Table 2).

For the female subgroup, two components were also extracted from the PCA. Unrotated factors accounted for 23.50 and $9.67 \%$ of the variance, respectively. The rotated solution showed that items $2,3,4,5$, $6,7,9,10,12,16$, and 20 were related to factor 1 , and items $8,14,15,20$, and 21 were related to factor 2. Cronbach's alpha coefficients for the subscales were 0.758 and 0.728 , respectively.

Two factors were also extracted for the male sub-group, accounting for 24.81 and $10.34 \%$ of the variance, respectively. The PCA solution suggested that items 2, 3, 5, 6, $7,9,10,12,14$, and 15 were related to factor 1 , and items $4,8,11,16,17,18$, and 20 were related to factor 2. Cronbach's alpha coefficients for the subscales were 0.806 and 0.701 , respectively.

\section{Discussion}

\section{BDI reliability and discriminant validity}

Previous psychometric studies of the BDI have reported that this instrument has acceptable reliability status (18). Review of internal consistency for the BDI ranges from 0.73 to 0.92 , with a mean of $0.86(7,18)$, with alpha coefficients of 0.86 and 0.81 for psychiatric and non-psychiatric populations, respectively (7). Therefore, our Cronbach's alpha coefficient of internal consistency of 0.82 demonstrated that the items of this Portuguese version of the BDI were homogeneous. The significant item-total correlation of most items indicated that they actually evaluated the same construct. The findings of low item-total correlation for the items loss of appetite, weight loss and indecisiveness are also consistent with previous international studies $(7,18)$.

A mean score of 10.0 was reported for a sample of Brazilian college students evaluated by the Portuguese version of the BDI (10). Indeed, for Chinese adolescents evaluated by the Chinese version of BDI, mean scores of 8.8-13.3 were found for women and 8.1-11.0 were found for men $(8,19)$. In a British sample of Chinese immigrants, mean BDI scores from 4.8 to 7.9 were reported for second-generation and first-generation Chinese immigrants, respectively, for the Chi- 
nese version of BDI (1). The present Portuguese version of BDI found lower, but acceptable, mean scores of 6.7, 6.6 and 6.9 for the total Chinese sample and for women and men, respectively. This finding may be the result of the sampling effect of selection, of the prevalence and severity of depression among Chinese individuals, of cultural influence (language semantics problem), and of measurement bias.

Most respondents were young bilingual adults (mean age of 24.9) and native Portuguese speakers (72\%). Those who were born in China or were first-generation immigrants had lived in Brazil for more than 20 years and had received Brazilian formal education. We recruited a convenience sample from a Protestant church attended by the Chinese community; therefore, a selection bias may have influenced the results. The attendees of church activities are considered to be healthier than the general population, and studies have indicated that religiousness is associated with fewer depressive symptoms $(20,21)$. For this immigrant population, the social network provided by religious meetings may work as a buffer against social isolation or deprivation. So, the cooperative respondents who returned the questionnaire may be healthier than those who do not attend church activities, thereby displaying less severe psychopathology.

The Chinese living in Taiwan $(22,23)$, Mainland China (24), and as immigrants in Los Angeles, CA, USA (2), present a lower prevalence of depression when compared with individuals reported in Western (25) and Brazilian studies (26). These findings are interesting in light of the suggestion of Kleinman (27), who viewed depression in Chinese culture as different, more somatic and less psychological. These cross-cultural studies suggest that culturally mediated values and views of symptoms influence the expression of psychiatric disorders, contributing to the lower expression of depressive symptoms in the Chinese population.
There is no major difference between European-influenced cultures in countries of English and Portuguese background (10), but probably there are marked differences on endorsement rates between Chinese respondents to the Portuguese version of the BDI and Brazilian respondents to the same measurement tool, although the former are well-adapted immigrants and native speakers of Portuguese.

Some affect-related constructs may differ cross-culturally in terms of the degree and nature of the affect. For example, suicide is regarded as a shameful behavior to talk about in Chinese society. Chinese individuals were less likely to communicate suicidal intent and some regard suicide as less effective to solve a problem. To deny or suppress this feared behavior, this term is more commonly expressed in a deficient or indirect mode in the Chinese language (15), e.g., "I will no longer live", "I don't want to live anymore". In contrast, the complaint of "fatigability" has better social acceptance among Chinese people, because being tired may just reflect the result of hard working or be a consequence of a modern lifestyle (28). Avoiding the reporting of suicidal intent may lead to an underestimate of the severity of depressive states, and over-complaining about fatigue may lead more to a diagnosis of neurasthenia or the Western equivalent, chronic fatigue syndrome. As such, the cultural sensitivity and appropriateness of the BDI's Portuguese items need to be determined for the Chinese Portuguese-speaking population in order to render the tool more culturally consonant for with the diverse characteristics of this sample.

Different from many Western (29-33) and even Brazilian studies (10), we were unable to demonstrate gender differences in scores of the Portuguese version of the BDI. Some researchers regard BDI as a genderbiased instrument (33), and past studies have reported that Chinese women are more likely to express their depressive symptoms than 
male respondents $(8,19)$. The scores of our bilingual respondents on the Portuguese BDI indicated that there was an obvious limitation in its application because there may be a difference in the style and the language of verbal expression of emotional and physical experiences between the Chinese and Brazilian culture (15). The ethnic Chinese respondents can understand most of the terms, but their semantic interpretation may be different. The absence of gender difference on item endorsement rates might have occurred because of lack of content reliability, with some items being semantically meaningless for the Chinese culture.

An alternative explanation for low scores and lack of gender difference on the BDI may be a measurement bias determined by the subjects' responses to the inventory. A measure deemed relatively easy for one group (e.g., Brazilian, Western) may be regarded as relatively difficult for another group (e.g., Chinese, Asian). When a measure is difficult, a floor effect can be observed, that is, many Chinese subjects obtained lower scores, close or corresponding to the minimum score possible. In this case, the measure was not sensitive to validly differentiate among subjects on the construct of interest (e.g., depression) and therefore failed to demonstrate gender differences in BDI scores. When there is a floor effect, and/or its counterpart ceiling effect, the score differences observed between two comparative groups are not meaningful.

Discriminant function analysis showed that the BDI was able to discriminate effectively between groups of depressed and nondepressed subjects in this Chinese population. We adopted a restrictive cut-off score above 20 as the criterion for considering the presence of depression (16), given that there was no concurrent diagnostic evaluation. The test yielded highly acceptable false-positive $(2.6 \%)$ and false-negative rates $(0 \%)$. The depression-specific items strongly predicted group membership (99\%), but the non-spe- cific items performed rather worse for the depressed subgroup (75\%).

Discriminant analysis revealed that the translated version of the BDI discriminated well depressive symptoms in Persian (34), Chinese (14), Spanish (35), and Portuguese speaking people (9). Meta-analyses of studies on the BDI content validity (36) revealed BDI validity in differentiating between depressed and non-depressed individuals. The BDI was also used to discriminate loneliness, stress and self-reported anxiety. Moreover, it discriminates between psychiatric and non-psychiatric patients, and produces relatively higher scores for patients with major depressive disorders compared with dysthymic disorders (37).

\section{Construct validity of BDI among ethnic Chinese}

Factor analysis of the BDI yielded inconsistent solutions across different studies. Main methodological differences, such as population characteristics, factor-extraction and factor-retention procedure, language version, and statistical approach, are aspects that might explain the variability of findings across many structural analyses of the BDI. For instance, reviews of factor analysis studies have revealed that the number of retained factors ranged from one to seven (7), including factors that reflect negative attitudes towards self, performance impairment and somatic disturbances, as well as a general factor of depression. However, two dimensions could be systematically extracted across the majority of the studies: the cognitive-affective factors and somatic factors. Recently, more robust confirmatory factor analyses have also supported this bidimensional view of the BDI structure $(38,39)$.

Factor analysis studies of the BDI in the Chinese population have described two-factor (13) and five-factor (12) solutions. Echoing previous findings and methodological problems, our factor analysis for the total 
sample suggested that two factors could be extracted, namely the cognitive-affective and somatic dimensions. For the first general factor, high loading was found on items such as guilt feelings, sense of failure, self-dislike, suicidal thoughts, lack of satisfaction, sense of punishment, and pessimism. For the somatic factor, the following items were important: fatigability, somatic preoccupation, distortion of body image, and work inhibition. Two non-Western factor analyses also yielded similar bidimensional structures for the BDI $(13,38)$. This cultural invariance of factorial structure demonstrates that the same underlying constructs measured by the BDI can be detected convergently with reasonable agreement across different samples and cultures.

Since sex differences may be related to differences in symptom profile, we carried out separate factor analyses by gender, which showed important differences in the expression of depressive symptoms between men and women at the item level, reflecting both component structure and symptom loading differences. Although the bidimensional structure of the present factor analysis was similar to that found for the Brazilian sample (10), many important differences concerning item loading indicated how expression of depressive symptoms is culturally mediated.

In factor 1 , male Chinese subjects showed higher loadings for sense of failure, guilt feelings, sense of punishment, self-dislike, pessimism, and suicidal thoughts. The highest loadings for factor 2 were on the items sleep disturbance, irritability, somatic preoccupation, loss of appetite, and fatigability. For Chinese women, the items that loaded the highest in factor 1 were suicidal wishes, lack of satisfaction, pessimism, self-dislike, crying spells, and sleep disturbances. The highest loadings for factor 2 were work inhibition, fatigability, distortion of body image, and somatic preoccupation.

The magnitude of the variance for each factor, as well as the internal consistency of both factors, was slightly higher for men. Generally speaking, depression-specific items loaded high in factor 1 , and somatoaffective or depression non-specific items in factor 2. One exception was the item sleep disturbance in the female subgroup that loaded high in the first general factor. It could also be seen that two items, sadness and loss of weight, did not contribute to any factor either in the total sample or in the female and male subgroups, differently from the Brazilian data (10). Although the cognitive-affective and somatic dimensions were present in both genders, factor 1 reflected more depression-specific items of cognitive distortion in men, whereas in women suicidal thoughts associated with depressive mood and low self-esteem prevailed. These differences between Chinese male and female expression of depressive symptoms remain to be confirmed in future studies on a clinical population with concurrent diagnoses established by clinical interviews and with a balanced distribution between men and women.

The cross-cultural and cross-linguistic utility of the BDI in a growing empirical knowledge base was demonstrated in this study. Our study with a limited sample size and specific cultural characteristics suggests that the BDI is a reliable self-reporting instrument for detecting depressive symptoms, with validity evidence concerning its use (discriminant and construct validity). Additionally, there was an interesting interplay between cultural factors and measurement bias, yielding a lower BDI score in this sample. In conclusion, this Portuguese version of the BDI can be regarded as useful to screen depression symptoms in the Chinese community. However, more culturally sensitive adaptation and semantical equivalence studies are recommended to further legitimate its validity (15).

This is a first attempt to validate the BDI for Brazilian Chinese immigrants. Bearing 
in mind the limitations of sample recruitment from a specific Chinese community, our results should not be prematurely extended to the entire Chinese community or Chinese clinical samples. Future cross-cultural studies of mental disorders will be greatly enhanced by the use of comparable screening instruments, with acceptable levels of reliability and criterion-validity compared to diagnostic evaluation $(4,40)$. The validation of this Portuguese version of the BDI (9-11) as a psychometrically sound and valid assessment measure will benefit the recipients of psychological services who are not native speakers. It should also facilitate the work of investigators conducting research on non-Western populations, as well as the comparison of research across countries and cultures.

\section{References}

1. Furham A \& Li YH (1993). The psychological adjustment of the Chinese community in Britain - a study of two generations. British Journal of Psychiatry, 162: 109-113.

2. Takeuchi DT, Chung RCY, Lin KM, Shen H, Kurasaki K, Chun CA \& Sue S (1998). Lifetime and twelve-month prevalence rates of major depressive episodes and dysthymia among Chinese Americans in Los Angeles. American Journal of Psychiatry, 155: 1407-1414.

3. Abbott MW, Wong S, Williams M, Au M \& Young W (1999). Chinese migrants' mental health and adjustment to life in New Zealand. Australian and New Zealand Journal of Psychiatry, 33: 13-21.

4. Yeung $A$, Neault N, Sonawalla S, Howarth S, Fava M \& Nierenberg AA (2002). Screening for major depression in Asian-Americans: a comparison of the Beck and the Chinese Depression Inventory. Acta Psychiatrica Scandinavica, 105: 252-257.

5. Wang YP, Gorenstein C \& Andrade LH (2004). Patterns of psychopathological manifestations among ethnic Chinese living in Brazil. European Archives of Psychiatry and Clinical Neuroscience, 254: 36-42.

6. Beck AT, Ward CH, Mendelson M, Mock J \& Erbaugh J (1961). An inventory for measuring depression. Archives of General Psychiatry, 4: 53-63.

7. Beck AT, Steer RA \& Garbin MG (1988). Psychometric properties of the Beck Depression Inventory. Twenty-five years of evaluation. Clinical Psychology Review, 8: 77-100.

8. Chan DW \& Tsoi MM (1984). The BDI and stimulus determinants of cognitive-related depression among Chinese college students. Cognitive Therapy and Research, 8: 501-508.

9. Gorenstein C \& Andrade L (1996). Validation of a Portuguese version of the Beck Depression Inventory and the State-Trait Anxiety Inventory in Brazilian subjects. Brazilian Journal of Medical and Biological Research, 29: 453-457.

10. Gorenstein C, Andrade L, Vieira Filho AHG, Tung TC \& Artes R (1999). Psychometric properties of the Portuguese version of the Beck Depression Inventory on Brazilian college students. Journal of Clinical Psychology, 55: 553-562.

11. Andrade L, Gorenstein C, Vieira Filho AH, Tung TC \& Artes R (2001). Psychometric properties of the Portuguese version of the state-trait anxiety inventory applied to college students: factor analysis and relation to the Beck Depression Inventory. Brazilian Journal of Medical and Biological Research, 34: 367-374.

12. Zheng YP, Wei L, Goa L, Zhang G \& Wong C (1988). Applicability of the Chinese Beck Depression Inventory. Comprehensive Psychiatry, 29: 484-489.
13. Shek DTL (1990). Reliability of factorial structure of the Chinese version of the Beck Depression Inventory. Journal of Clinical Psychology, 46: 35-42.

14. Shek DTL (1991). What does the Chinese version of the Beck Depression Inventory measure in Chinese students - general psychopathology or depression? Journal of Clinical Psychology, 47: 381-390.

15. Zheng YP \& Lin KM (1991). Comparison of the Chinese Depression Inventory and the Chinese version of the Beck Depression Inventory. Acta Psychiatrica Scandinavica, 84: 531-536.

16. Kendall PC, Hollon SD, Beck AT, Hammen CL \& Ingram RE (1987). Issues and recommendations regarding use of the Beck Depression Inventory. Cognitive Therapy and Research, 11: 289-299.

17. Salamero M, Marcos T, Gutiérrez F \& Rebull E (1994). Factor study of the BDI in pregnant women. Psychological Medicine, 24: 10311035.

18. Beck AT \& Steer RA (1984). Internal consistencies of the original and revised Beck Depression Inventory. Journal of Clinical Psychology, 40: 1365-1367.

19. Stewart $S M$, Betson $C L$, Lam TH, Chung SF, Ho HH \& Chung TCF (1999). The correlates of depressed mood in adolescents in Hong Kong. Journal of Adolescent Health, 25: 27-34.

20. Powell LH, Shahabi L \& Thorensen CE (2003). Religion and spirituality. Linkages to physical health. American Psychologist, 58: 36-52.

21. Koenig HG (2001). Religion and medicine II: religion, mental health, and related behaviors. International Journal of Psychiatry and Medicine, 31: 97-109.

22. Hwu HG, Yeh EK \& Chang LY (1989). Prevalence of psychiatric disorders in Taiwan defined by the Chinese diagnostic interview schedule. Acta Psychiatrica Scandinavica, 79: 136-147.

23. Cheng TA (1988). A community study of minor psychiatric morbidity in Taiwan. Psychological Medicine, 18: 953-968.

24. Cooper JE \& Sartorius N (1996). Mental Disorder in China - Results of the National Epidemiological Survey in 12 Areas. Gaskell, Glasgow, Scotland.

25. Andrade L, Caraveo-Anduaga JJ, Berglund P et al. (2003). The epidemiology of major depressive episodes: results from the International Consortium of Psychiatric Epidemiology (ICPE) Surveys. International Journal of Methods in Psychiatric Research, 12: 3-21.

26. Andrade L, Walters EE, Gentil V \& Laurenti R (2002). Prevalence of ICD-10 mental disorders in a catchment area in the city of São Paulo, Brazil. Social Psychiatry and Psychiatric Epidemiology, 37: 316-325. 
27. Kleinman A (1982). Neurasthenia and depression: a study of somatization and culture. Culture, Medicine and Psychiatry, 6: 117-190.

28. Lee S \& Wong KC (1995). Rethinking neurasthenia: the illness concepts of shenjing shuairuo among Chinese undergraduates in Hong Kong. Culture, Medicine and Psychiatry, 19: 91-111.

29. Hammen CL \& Padesky CA (1977). Sex difference in the expression of depressive responses on the Beck Depression Inventory. Journal of Abnormal Psychology, 86: 609-614.

30. Baron P \& Campbell TL (1993). Gender differences in the expression of depressive symptoms in middle adolescents: an extension of earlier findings. Adolescence, 28: 903-911.

31. Byrne DG (1981). Sex differences in the reporting of symptoms of depression in the general population. British Journal of Clinical Psychology, 20: 83-92.

32. Steer RA, Beck AT \& Brown G (1989). Sex differences on the revised Beck Depression Inventory for outpatients with affective disorders. Journal of Personality Assessment, 53: 693-702.

33. Salokangas RK, Vaahtera K, Pacriev S, Sohlman B \& Lehtinen V (2002). Gender differences in depressive symptoms. An artefact caused by measurement instruments? Journal of Affective Disorders, 68: 215-220.

34. Hojat M, Shapurian R \& Mehrya AH (1986). Psychometric properties of a Persian version of the short form of the Beck Depression Inventory for Iranian college students. Psychological Reports, 59: 331-338.

35. Bonicatto S, Dew AM \& Soria JJ (1998). Analysis of the psychometric properties of the Spanish version of the Beck Depression Inventory in Argentina. Psychiatry Research, 79: 277-285.

36. Richter P, Werner J, Heerlien A, Kraus A \& Sauer H (1998). On the validity of the Beck Depression Inventory: a review. Psychopathology, 31: 160-168.

37. Steer RA, Beck AT, Riskind JH \& Brown G (1986). Differentiation of depressive disorders from generalized anxiety by the Beck Depression Inventory. Journal of Clinical Psychology, 42: 475-478.

38. Kojima M, Furukawa TA, Takahashi H, Kawai M, Nagaya $T$ \& Tokudome S (2002). Cross-cultural validation of the Beck Depression Inventory-II in Japan. Psychiatry Research, 110: 291-299.

39. Steer RA, Ball R, Ranieri WF \& Beck AT (1999). Dimensions of the Beck Depression Inventory-II in clinically depressed outpatients. Journal of Clinical Psychology, 55: 117-128.

40. Lasa L, Ayuso-Mateos JL, Vázquez-Barquero JL, Díez-Manrique FJ \& Dowrick CF (2000). The use of the Beck Depression Inventory to screen for depression in the general population: a preliminary analysis. Journal of Affective Disorders, 57: 261-265. 